\title{
BMJ Open Internet-based patient- primary care physician-cardiologist integrated management model of hypertension in China: study protocol for a multicentre randomised controlled trial
}

\author{
Runyu Ye, Rufeng Shi, Kai Liu, Xin Zhang, Si Wang, Hang Liao, Xinran Li, \\ Qiling Gou, Xi Rong, Zhipeng Zhang, Changqiang Yang, Xiangyu Yang, \\ Xiaoping Chen
}

To cite: Ye R, Shi R, Liu K, et al. Internet-based patient- primary care physician-cardiologist integrated management model of hypertension in China: study protocol for a multicentre randomised controlled trial. BMJ Open 2020;10:e039447. doi:10.1136/ bmjopen-2020-039447

- Prepublication history and additional material for this paper are available online. To view these files, please visit the journal online (http://dx.doi. org/10.1136/bmjopen-2020039447).

RY and RS contributed equally.

Received 15 April 2020

Revised 31 August 2020

Accepted 02 September 2020

D Check for updates

(c) Author(s) (or their employer(s)) 2020. Re-use permitted under CC BY-NC. No commercial re-use. See rights and permissions. Published by BMJ.

Cardiology, West China Hospital of Sichuan University, Chengdu, Sichaun, China

Correspondence to

Dr Xiaoping Chen;

xiaopingchen15@126.com

\section{ABSTRACT}

Introduction The control rate of hypertension is low in China, especially in rural, western and minority areas. This is related to poor medical skills among physicians in primary care institutions and low levels of trust among patients. However, primary healthcare institutions are the main battleground for the prevention and treatment of hypertension. It is worth exploring how to most effectively integrate patients, primary care physicians and cardiologists in tertiary hospitals, to build a longterm mechanism for the prevention and treatment of hypertension. In this study, we aim to evaluate the clinical effectiveness and conduct a health economic evaluation of an internet-based patient-primary care physician-cardiologist integrated management model of hypertension in areas of China with different socioeconomic levels.

Methods and analysis This is a 12-month, multicentre, randomised controlled trial involving patients with hypertension in urban communities and rural areas of Sichuan Province, China. Each primary healthcare institution will cooperate with their tertiary hospital through the Red Shine Chronic Disease Management System (RSCDMS). Patients will be randomly assigned 1:1 to two groups: (1) a traditional care group; (2) an intervention group in which primary care physicians and cardiologists can share patient data and manage patients together through the RSCDMS. Patients can upload their blood pressure (BP) values and communicate with physicians using the system. The primary outcome is the change in systolic BP over a 12-month period. Secondary outcomes are changes in diastolic BP, BP control rate, values of 24-hour ambulatory BP monitoring, difference in costeffectiveness between the groups, patient satisfaction, medication adherence and home BP monitoring compliance. All data will be recorded and stored in the RSCDMS and analysed using IBM SPSS V.26.0.

Ethics and dissemination This study has been approved by the Biomedical Research Ethics Committee of the West China Hospital of Sichuan University in Sichuan, China (No. 2020-148). Written informed consent will be obtained from all participants. The results of this study will be
Strengths and limitations of this study

This 12-month, multicentre, randomised controlled trial will explore the effectiveness of a comprehensive hypertension management model that incorporates telemedicine technologies.

- Each primary healthcare institution will cooperate with their tertiary hospital through the Red Shine Chronic Disease Management System.

- The Red Shine Chronic Disease Management System will be used as a tool to integrate patients, primary care physicians and cardiologists.

- This study will be conducted in southwest China, including patients with hypertension in urban communities and rural areas of Sichuan Province, to test the clinical effectiveness and cost-effectiveness of the model in areas with different socioeconomic levels.

- The study will only be conducted in southwest China, whether this model can be generalised to other regions remains uncertain.

disseminated to the public through academic conferences and peer-reviewed journals.

Trial registration number ChiCTR2000030677.

\section{INTRODUCTION}

Hypertension is a major risk factor for cardiovascular disease. The latest survey suggests that the prevalence of hypertension in China is $27.9 \%$, with just a $16.8 \%$ control rate. ${ }^{2}$ The current situation of hypertension is at a rather low level, especially in rural, western and minority areas, causing a heavy disease burden. ${ }^{13}$ Studies have shown that actively preventing and controlling hypertension is a core strategy in restraining the development of cardiovascular and cerebrovascular diseases. ${ }^{45}$ Nearly $90 \%$ of China's population with hypertension are distributed throughout 
primary hospitals, that is, urban and rural community health centres. ${ }^{67}$ Therefore, primary hospitals are the main battleground for the prevention and treatment of hypertension. ${ }^{89}$ At present, the traditional management model requires that patients be followed up in primary hospitals according to their blood pressure (BP) level. ${ }^{2}$ However, patients have low levels of trust in the medical facilities and treatment in primary hospitals. Thus, the treatment measures and lifestyle advice received from primary care physicians (PCPs) are not effectively implemented and maintained by patients. Routine follow-ups are not carried out in time, affecting BP control. ${ }^{7} 10$ Therefore, the current situation of hypertension management in primary hospitals must be improved.

Previous studies have shown that patients are more satisfied with diagnoses and treatment provided by doctors in tertiary hospitals. ${ }^{11}{ }^{12}$ With the participation of specialists in community hypertension management, the control of BP is superior to that under traditional care management. ${ }^{13} 14$ Although China is working hard to strengthen the training of PCPs to improve their professional level, the integration of professional knowledge is a gradual and long-term process. PCPs might lack the ability to deal with some patients who have poor BP control. Therefore, it is feasible for specialists to go into the community to provide consultations. However, the busy work schedule of these specialists renders the long-term operation of this management model unfeasible.

Based on these problems with the traditional hypertension management model, it is worth exploring how to most effectively integrate patients, PCPs and cardiologists in tertiary hospitals, to build a long-term mechanism for the prevention and treatment of hypertension.

With the advent of the information era, studies have focused on the effects of telemedicine on chronic disease management. ${ }^{15-17}$ Guidelines recommend that telehealth strategies can be a useful method to improve hypertension control by implementing remote BP recordings, e-prescriptions, health education and e-counselling. ${ }^{15}$ These methods could improve patients' access and participation in hypertension management. Using e-counselling strategies is an effective way to improve $\mathrm{BP}$, which led to a decrease of systolic BP (SBP) by $10.1 \mathrm{~mm} \mathrm{Hg}$ compared with $6.0 \mathrm{~mm} \mathrm{Hg}$ in the control group of one study. ${ }^{17}$ Until now, nearly 200 smartphone applications have been developed for hypertension management. ${ }^{18}$ However, these lack validation of their effectiveness and the involvement of healthcare professionals in the appdevelopment process. In addition, none of these apps has established an integrated link with high-quality medical resources among patients, PCPs and specialists. Therefore, these apps cannot be effectively implemented in hypertension management.

Based on the above situation, our team developed an internet-based hypertension management system, the Red Shine Chronic Disease Management System (RSCDMS), with the cooperation of cardiologists and software technicians. Through this system, patients can receive effective management by physicians under the guidance of specialists, and PCPs can improve their skills in practice. In this trial, we aim to evaluate the clinical effectiveness and conduct a health economic evaluation of this internet-based three-level integrated management model of hypertension in areas of China with different socioeconomic levels.

\section{METHODS AND ANALYSIS \\ Study design}

This study is designed as a 12-month multicentre trial among patients with hypertension in urban communities and rural areas. The study design will be described in accordance with the Standard Protocol Items: Recommendations for Interventional Trials 2013 checklist ${ }^{19} 20$ (online supplemental material 1 and table 1).

\section{Patient and public involvement}

This trial is designed to evaluate the clinical effectiveness and health economics of an internet-based patientprimary care physician-cardiologist (PPC) integrated management model of hypertension in areas of China with different socioeconomic levels. The RSCDMS will be used as a tool to execute the management process. Participants will be recruited from six primary hospitals managed by three tertiary hospitals. However, they will not be directly involved in the design, recruitment or execution of the study. After the trial is completed, the results of this study will be disseminated to the public via conference presentations and peer-reviewed journals. When the manuscript is published, we will summarise the results and text them to all trial participants in a brief message.

\section{Study outcomes}

Primary outcome

The primary outcome is the difference between the last mean office SBP value and baseline office SBP after 12 months of intervention.

\section{Secondary outcomes}

The secondary outcomes are:

1. Changes in mean office diastolic BP from baseline.

2. Office BP control rate (controlled BP is defined as $\mathrm{BP}<140 / 90 \mathrm{~mm} \mathrm{Hg}$ or $<130 / 80 \mathrm{~mm} \mathrm{Hg}$ (for patients with diabetes or renal diseases) following the Chinese guidelines $^{2}$ ).

3. Changes related to body metabolism (weight, waist circumference, blood lipid levels and blood glucose level).

4. Changes from baseline in values of 24-hour ambulatory blood pressure monitoring (ABPM): mean 24-hour, daytime and night-time BP.

5. Difference in cost-effectiveness between groups.

6. Changes from baseline in patient satisfaction evaluated using the European Task Force on Patient Evaluations of General Practice. ${ }^{21}$ 
Table 1 Study timelines overview of all visits and tests scheduled in the trial

\section{Study period}

\begin{tabular}{|c|c|c|c|c|c|}
\hline \multirow[b]{2}{*}{ Timepoint } & \multirow{2}{*}{$\begin{array}{l}\text { Enrolment } \\
\text { Visit } 0 \\
\text { week } 1\end{array}$} & \multicolumn{4}{|l|}{ Follow-up } \\
\hline & & $\begin{array}{l}\text { Visit } 1 \\
\text { month } 1\end{array}$ & $\begin{array}{l}\text { Visit } 2 \\
\text { month } 3\end{array}$ & $\begin{array}{l}\text { Visit } 3 \\
\text { month } 6\end{array}$ & $\begin{array}{l}\text { Visit } 4 \\
\text { month } 12\end{array}$ \\
\hline Eligibility screen & $x$ & & & & \\
\hline Informed consent & $x$ & & & & \\
\hline \multicolumn{6}{|l|}{ Interventions } \\
\hline Control group & & $x$ & $x$ & $x$ & $x$ \\
\hline Intervention group & & $x$ & $x$ & $x$ & $x$ \\
\hline \multicolumn{6}{|l|}{ Assessments } \\
\hline Medical history & $x$ & & & & \\
\hline \multicolumn{6}{|l|}{ Lab tests } \\
\hline Blood routine & $x$ & & & $x$ & $x$ \\
\hline Serum lipids & $x$ & & & $x$ & $x$ \\
\hline Hepatic function & $x$ & & & $x$ & $x$ \\
\hline Renal function & $x$ & & & $x$ & $x$ \\
\hline FPG & $x$ & & & $x$ & $x$ \\
\hline Urine routine & $x$ & & & $x$ & $x$ \\
\hline ABPM & $x$ & $x$ & & & $x$ \\
\hline
\end{tabular}

Hepatic function refers to total bilirubin; alanine aminotransferase; aspartate aminotransferase; gamma glutamine transferase; alkaline phosphatase and albumin. Renal function refers to serum creatinine; blood urea nitrogen and blood uric acid.

ABPM, 24-hour ambulatory blood pressure monitoring; BMQ, Brief Medication Questionnaire; BP, blood pressure; EQ-5D, EuroQol fivedimensional; EUROPEP, European Task Force on Patient Evaluations of General Practice; FPG, fasting plasma glucose.

7. Changes from baseline in the antihypertensive medication adherence evaluated by a derived version of the Brief Medication Questionnaire. $^{22}$

8. Home BP monitoring compliance including the frequency of home BP measurements per month (derived from RSCDMS or logbook), and the proportion of months when a home BP reading is obtained. ${ }^{23}$

\section{Eligibility criteria}

Inclusion criteria

1. Age between 18 and 80 years.

2. Newly diagnosed hypertension, or with a history of hypertension and uncontrolled $\mathrm{BP}$ in the past 3 months $(\geq 140 / 90 \mathrm{~mm} \mathrm{Hg}$ or $\geq 130 / 80 \mathrm{~mm} \mathrm{Hg}$ if the patient has diabetes/renal disease), currently taking or about to take antihypertensive drugs.

3. Able to use a smartphone.

Exclusion criteria

1. Secondary hypertension.

2. Heart failure.

3. Hospitalised for acute myocardial infarction within the past 6 months.

4. Severe hepatic or renal disease (serum aspartate aminotransferase/alanine aminotransferase levels three times above the normal limit or glomerular filtration rate $<30 \mathrm{~mL} / \mathrm{min}$ ) or serum creatinine $>221 \mu \mathrm{mol} / \mathrm{L}$ ).

5. Planning to undergo surgery within 6 months. 
6. History of malignant tumours.

7. Cognitive dysfunction or unable to take care of self.

8. Participating in other clinical trials.

\section{Study setting and recruitment}

Participants will be recruited from six primary hospitals managed by three tertiary hospitals in Sichuan Province, China, and be randomised according to three areas. According to their economic development level, three areas will be included:

1. Economically and medically developed areas in Sichuan Province.

2. Regions with average socioeconomic and medical resource levels.

3. Economically and medically underdeveloped areas in Sichuan Province.

Potential participants will be identified by screening electronic medical records in primary hospitals, roughly following the eligibility criteria. Eligible participants are then to be screened by telephone to determine their interest in participation, experience using smart phones and willingness to sign a consent form (online supplemental material 2). Those who remain eligible will be invited to their primary hospital to attend a screening session with PCPs and cardiologists. Eligible patients will be randomised to the control or the intervention group. Participants in the intervention group will be instructed in how to use the WeChat applet during management. The recruitment process is expected to take 5 months to complete, from May to September 2020.

\section{Interventions}

Participants in each primary hospital will be divided into two groups. Group 1 will receive traditional care that complies with China's Hypertension Prevention and Treatment Guideline 2018, ${ }^{2}$ but PCPs will not intervene in participants' health-seeking behaviour. Group 2 will additionally receive internet-based PPC-integrated management, which will include coordinated management executed by PCPs and cardiologists (figure 1).

Group 1: control group, traditional care model

After recruitment, group 1 will be managed by their PCPs. Patients will receive the usual management and follow-up

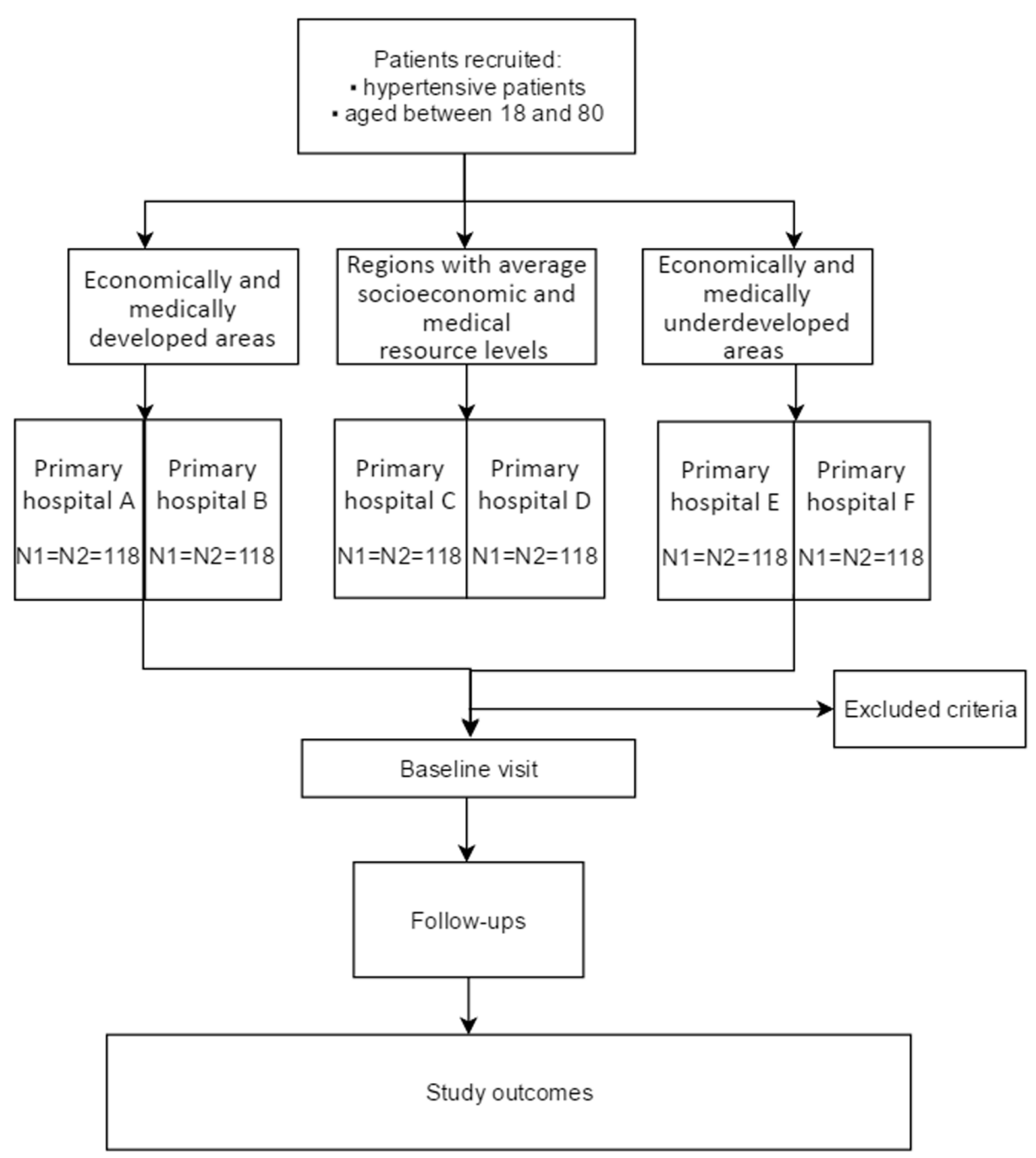

Figure 1 Study design and flow diagram. 


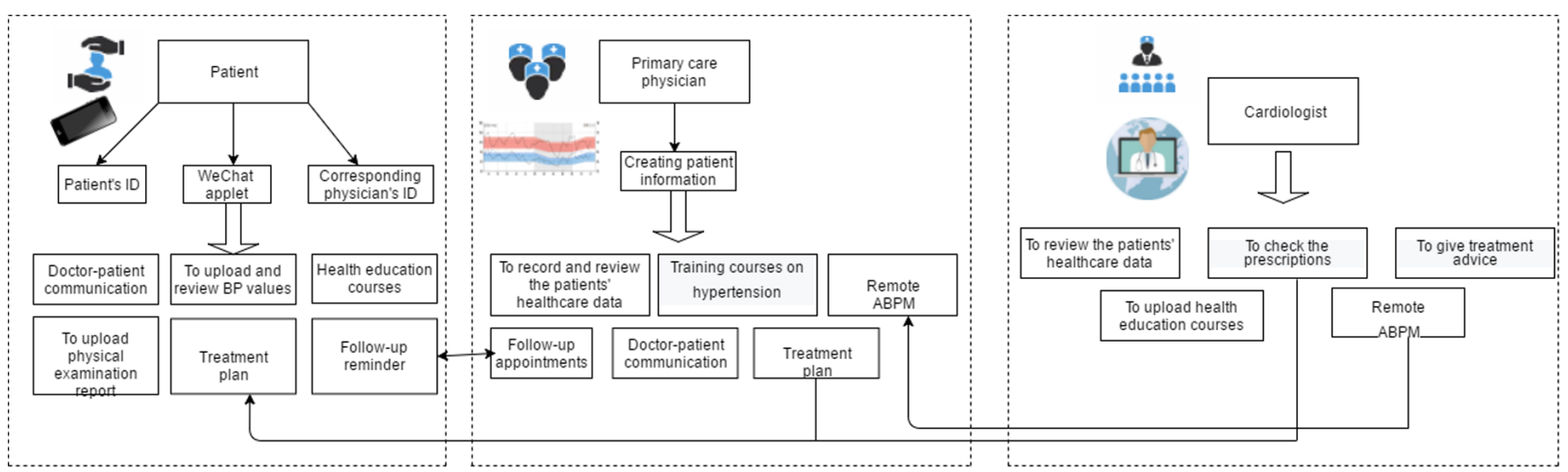

Figure 2 Function of the Red Shine Chronic Disease Management System. ABPM, 24-hour ambulatory blood pressure monitoring.

in compliance with the national guideline. This model includes designated follow-ups by PCPs once every 2-4 weeks if $\mathrm{BP}$ is not controlled, and once every 3 months if $\mathrm{BP}$ is within the normal range. Patients will be encouraged to record their home BP readings using a logbook and to present the results during their visit. Physicians will provide medication prescriptions and lifestyle advice, as suggested in the guidelines. Follow-ups are to be conducted through outpatient visits and by telephone.

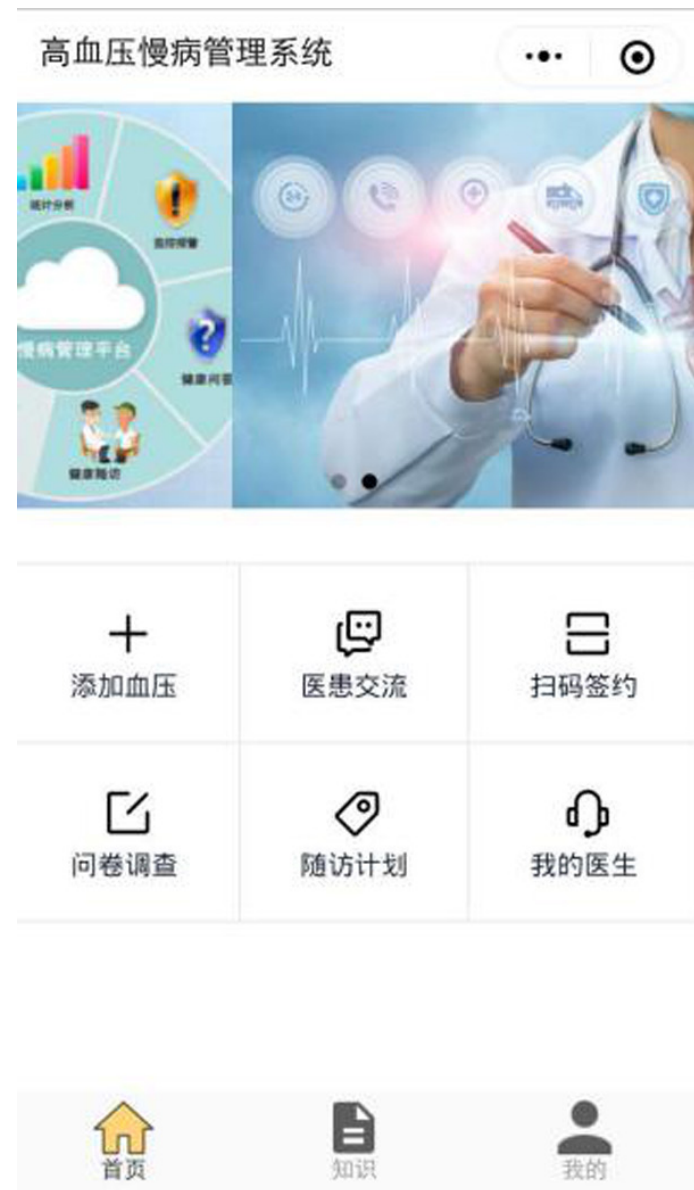

Figure 3 The screenshot for the module of the patients.
Group 2: intervention group, internet-based PPC-integrated management model

Patients in this group will be managed by PCPs together with cardiologists using the RSCDMS. The system consists of three modules (figures 2-4).

1. The first module is a WeChat applet for patients to upload their BP values, communicate with PCPs about their condition and browse through health education courses uploaded by cardiologists. This applet will also serve as a reminder for patients to complete their follow-up appointments on time.

2. The second module is a website for PCPs to record and review patients' medical data, provide treatment plans, make follow-up appointments, communicate with patients and upload ABPM data.

3. The third module is a website for cardiologists to review patients' medical data, check prescriptions provided by PCPs and give suggestions, upload health education information using short videos or popular science articles, and analyse the ABPM data.

Cardiologists will review patients' health data and provide treatment suggestions within 2 days after the patient is enrolled and during each follow-up visit. If the patient's condition worsens or hypertension complications occur, the system can be used to coordinate referral to a tertiary hospital.

The RSCDMS is used as a tool to integrate patients, PCPs and cardiologists. Using the three functional modules, synchronous online management and a real-time hierarchical medical system can be delivered by PCPs and cardiologists in tertiary hospitals.

\section{Data collection and management}

The processes of data collection, storage and delivery are achieved through the RSCDMS (http://39.105.211.76:3000/doctor/index). Researchers will manually record the medical information in this internet-based system. Data from the same patient will be entered by one investigator and verified by another investigator to avoid missing, duplicate and erroneous data. A trial inspector will visit each site regularly to check the 


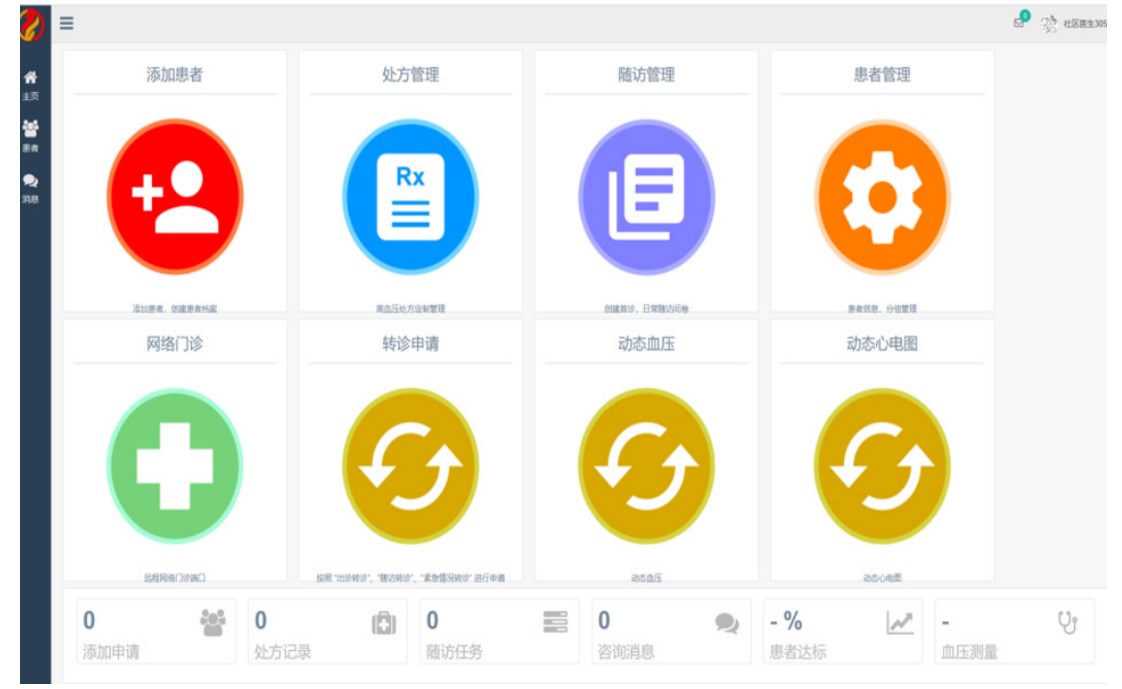

Figure 4 The screenshot for the website of the physicians.

research progress and ensure that the trial strictly adheres to the protocol.

Venous blood will be drawn in the morning after an 8-hour fast, and the levels of blood glucose, liver enzymes, creatinine and the serum levels of lipids including triglycerides, total cholesterol, low-density lipoprotein (LDL) cholesterol and high-density lipoprotein cholesterol will be evaluated. Office BP will be measured using a validated Omron upper-arm electronic sphygmomanometer (HBP-9020; Omron Co., Dalian, China), according to the 2018 European Society of Cardiology/European Society of Hypertension (ESH) guidelines. ${ }^{24}$ Two or three readings of the heart rate and $\mathrm{BP}$ are to be taken and the mean recorded. ${ }^{24}$ Home BP monitoring will be carried out by the patients themselves using their own upper-arm electronic sphygmomanometer (meeting criteria of the Association for the Advancement of Medical Instrumentation/ESH/International Organization for Standardization), ${ }^{25}$ and $\mathrm{BP}$ values will be manually entered into the WeChat applet. Patients will be taught when and how to conduct home BP monitoring by PCPs and cardiologists during the screening sessions, according to the guidelines. $^{25}$

Adverse events include syncope, falls, hypotension, coronary heart disease, stroke and heart failure. Cost information will be collected during each follow-up, including direct costs and indirect costs. Direct costs refer to direct medical costs (antihypertensive drugs, laboratory tests, examinations during follow-up and treatment, and hospitalisation owing to cardiovascular, cerebrovascular and renal events) and direct non-medical costs (transportation, meals and other expenses that arise owing to illness). Indirect costs refer to loss-of-life costs and economic losses caused by illness and missed work.

All patients, PCPs and cardiologists will have a unique account number and password. The account number of each patient corresponds to a PCP and a cardiologist, and the account number of each cardiologist corresponds to the account number of two to three PCPs. The research staff is only provided with read permission for the database.

\section{Study timeline}

A diagram summarising the timeline is presented in table 1 .

\section{Randomisation}

Participants will be sequentially allocated to the intervention or control group with a 1:1 ratio using centrestratified block randomisation. The random allocation sequence will be generated by a statistician prior to study initiation using SAS V.9.4. To limit selection bias, the allocation sequence is to be concealed from all study researchers in well-sealed, opaque envelopes until the interventions are assigned. Owing to the nature of the study, this will be an open-label trial for all participating patients, physicians and inspectors at each site.

\section{Sample size calculation}

Sample size calculation is based on the primary outcome, namely, differences in the change of SBP between the two groups at 12 months. This study is powered to detect a difference of at least $10 \mathrm{~mm} \mathrm{Hg}$ in SBP. ${ }^{26} 27$ We assume that the SD for the difference in SBP from baseline to 12 months is $10 \mathrm{~mm} \mathrm{Hg}{ }^{28}{ }^{29}$ Using a two-sample comparison of the mean change between group 1 and group 2, a sample size of 100 patients per group is required to have more than $99 \%$ power. Accounting for an estimated drop-out rate of $15 \%$, the sample size is adjusted to 118 participants per group for each site (6 sites, 2 groups per site, 1416 in total). To reduce potential drop-out, if a patient chooses to discontinue the trial, we will contact them and determine their reasons for dropping out. According to the situation, we will attempt to persuade the patient to continue their participation in the study.

\section{Statistical analysis}

Statistical analysis will be performed using IBM SPSS V.26.0. Baseline data will be presented using descriptive 
statistics (mean \pm SD for normally distributed continuous variables, median and IQR for non-normally distributed continuous variables, and frequency and percentage for categorical variables). For comparisons between two groups, a t-test or rank-sum test will be used for quantitative variables, and the $\mathrm{X}^{2}$ test or Fisher's exact test will be used for qualitative variables. The primary outcome of this study, differences in the change of SBP, will be estimated using a safety set in which all randomised participants with data for at least one follow-up visit will be analysed. A covariance analysis model will be fitted for the primary outcome, adjusting the baseline using the following covariates, as appropriate: baseline SBP, age, sex, body mass index, drinking status, smoking status, fasting blood glucose, total cholesterol and LDL cholesterol. Only those covariates found to be statistically significant for the outcomes will be selected as explanatory variables for inclusion in the final model. Data will be presented as adjusted mean difference (95\% CI) and corresponding $\mathrm{p}$ values. We will conduct further analysis to evaluate secondary outcomes using the identical methods described above for the primary outcome analysis. For cost-effectiveness analysis, $\mathrm{R}$ software will be used for construction of a decision tree (The R Project for Statistical Computing, Vienna, Austria).

\section{Ethics and dissemination}

This study has been approved by the Biomedical Research Ethics Committee of the West China Hospital of Sichuan University in Sichuan, China (No. 2020-148). Written informed consent will be obtained from all participants. The results of this study will be disseminated to the public through academic conferences and peer-reviewed journals.

\section{DISCUSSION}

The disease burden of hypertension in China is experiencing a growing trend, with a large number of patients who have hypertension and limited physicians in primary hospitals. Tertiary hospitals in China are overcrowded and usually dealing with complicated diseases, leaving physicians less time to manage patients with hypertension. The key to better prevention and control of hypertension lies in the primary hospitals as they are more easily accessible. However, the limited number of PCPs makes patient management inefficient in routine visits.

Recent studies have shown that the use of comprehensive multidimensional hypertension management among low-income populations with relatively limited medical resources can significantly improve the BP control rate. ${ }^{28-30}$ In China, implementing a hierarchical medical system and improving the hypertension management capacity of physicians in areas with underdeveloped medical resources is especially important. ${ }^{31}{ }^{32}$ However, the busy schedules of PCPs in primary hospitals, as well as their limited capacity to deal with complex diseases, lead to a hierarchical medical system becoming an armchair strategy. ${ }^{33} 34$

Meta-analyses have shown that telemedicine can help improve the BP control rate and cardiovascular event outcomes. ${ }^{16} 3536$ It can also compensate for physical distance between patients in remote areas and major medical institutions. In addition to these benefits, telemedicine can provide considerable economic benefits. ${ }^{37}$ Data from 25182 teleconsultation visits suggest that a total of US\$9 175 132, and 1876146 hours (213.1 years) in chronic disease management have been saved with the use of telemedicine in low-income and middle-income countries.

In the context of a national hierarchical medical system, a comprehensive hypertension management model based on telemedicine is worth exploring for use in urban communities and remote rural areas. The present model is designed to fit within the current conditions in China's community healthcare system, to support the hypothesis that extending the role of specialists and making them more available for community healthcare services can enhance the effectiveness of patient management. Previous studies have demonstrated that under the guidance of specialists, $\mathrm{BP}$ control can be improved in primary care institutions. ${ }^{1314}$ However, in these studies, researchers have provided consultation in the community, which necessitates that specialists travel from their workplace to communities. This method is effective but also consumes time and energy. Moreover, this approach could go on for a time, but a high rate of BP control cannot be sustained in the long run. Ours is the first multicentre study focusing on use of the internet to connect patients, PCPs and cardiologists in different regions and with differing socioeconomic levels. This study will shed light on web-based management of hypertension in China and provide future guidance on the implementation of a hierarchical medical system.

In addition to assessing the efficacy of the above intervention, this study will have several other impacts. First, through use of this internet system, the difficulties with visiting a doctor in a tertiary hospital experienced by residents in the community and in remote areas could be resolved by reducing the number of times patients must travel to and from hospitals, in addition to improving patient data management. Second, the patient data can be shared between the primary healthcare institution and tertiary hospital in real time, improving the management efficiency. Third, we can determine whether the internetbased management model is suitable for different kinds of institutions and regions. Fourth, physicians in underdeveloped areas can learn from specialists through cooperation, improving medical standards in practice.

There are also some limitations in our study. First, our study will only be conducted in southwest China, although we will include regions with different socioeconomic levels. Thus, whether this model can be generalised to other regions remains uncertain. Second, the sample size is relatively small considering the actual 
number of patients with hypertension in clinical practice. It is therefore necessary to explore whether this model can function when the number of patients increases to the normal levels in clinical practice as well as in the long term. Third, the home BP monitoring values will be manually uploaded to the WeChat applet by patients rather than being automatically uploaded via Bluetooth. Currently, many electronic sphygmomanometers lack the ability to transmit BP data automatically. We aim to test this internet-based model over the long term, such that participants will be able to continue to use their own electronic sphygmomanometers and record their BP using the WeChat applet. Fourth, we did not provide BP monitors for patients as home BP monitoring values are not an outcome measure. Thus, the BP monitors used by participants will differ and the uploaded BP values may lack consistency. However, we will ask patients to regularly calibrate their BP monitors, to minimise this difference.

Acknowledgements We would like to thank Chengdu Saibaili Medical Technology Co. for co-developing the software with our research team.

Contributors $\mathrm{XC}$ is the principal investigator of this study. RY and RS contributed equally to the article, who developed the study concept and wrote the manuscript. $\mathrm{KL}$ and XZ participated in the development of the Red Shine Chronic Disease Management System. SW and HL designed the method for statistic analysis. RY, $\mathrm{XL}, \mathrm{QG}, \mathrm{XR}, \mathrm{ZZ}, \mathrm{CY}$ and $\mathrm{XY}$ participated in the modification of the system and the manuscript. All authors reviewed and approved the final manuscript.

Funding This work is supported by Science and Technology Pillar Programs in Sichuan Province (Grant number 2017SZ0008).

Competing interests None declared.

Patient consent for publication Not required.

Provenance and peer review Not commissioned; externally peer reviewed.

Supplemental material This content has been supplied by the author(s). It has not been vetted by BMJ Publishing Group Limited (BMJ) and may not have been peer-reviewed. Any opinions or recommendations discussed are solely those of the author(s) and are not endorsed by BMJ. BMJ disclaims all liability and responsibility arising from any reliance placed on the content. Where the content includes any translated material, BMJ does not warrant the accuracy and reliability of the translations (including but not limited to local regulations, clinical guidelines, terminology, drug names and drug dosages), and is not responsible for any error and/or omissions arising from translation and adaptation or otherwise.

Open access This is an open access article distributed in accordance with the Creative Commons Attribution Non Commercial (CC BY-NC 4.0) license, which permits others to distribute, remix, adapt, build upon this work non-commercially, and license their derivative works on different terms, provided the original work is properly cited, appropriate credit is given, any changes made indicated, and the use is non-commercial. See: http://creativecommons.org/licenses/by-nc/4.0/.

ORCID iD

Xiaoping Chen http://orcid.org/0000-0001-7714-3403

\section{REFERENCES}

1 Wang Z, Chen Z, Zhang L, et al. Status of hypertension in China: results from the China hypertension survey, 2012-2015. Circulation 2018;137:2344-56.

2 Revision Committee of guidelines on the management of hypertension in China. 2018 Chinese guidelines for the management of hypertension. ZhongGuo Xin Xue Guan Za Zhi 2019;24:24-56.

3 Lu J, Lu Y, Wang X, et al. Prevalence, awareness, treatment, and control of hypertension in China: data from 1.7 million adults in a population-based screening study (China peace million persons project). Lancet 2017;390:2549-58.

4 Staessen JA, Wang JG, Thijs L, et al. Cardiovascular protection and blood pressure reduction: a meta-analysis. Lancet 2001;358:1305-15.
5 Staessen JA, Gasowski J, Wang JG, et al. Risks of untreated and treated isolated systolic hypertension in the elderly: meta-analysis of outcome trials. Lancet 2000;355:865-72.

6 Wang W, Wang ZW, Chen WW. The key of prevention and treatment of hypertension in China lies at the grass-roots level. Zhonghua Gao Xue Ya Za Zhi 2010;18:501-3.

7 Zou Y, Zhang X, Hao Y, et al. General practitioners versus other physicians in the quality of primary care: a cross-sectional study in Guangdong Province, China. BMC Fam Pract 2015;16:134.

8 Lu C-H, Tang S-T, Lei Y-X, et al. Community-Based interventions in hypertensive patients: a comparison of three health education strategies. BMC Public Health 2015;15:33.

9 Kaczorowski J, Del Grande C, Nadeau-Grenier V. Community-Based programs to improve prevention and management of hypertension: recent Canadian experiences, challenges, and opportunities. Can J Cardiol 2013;29:571-8.

10 Kong X, Yang Y. The current status and challenges of community general practitioner system building in China. QJM 2015;108:89-91.

11 YAN HL, Xiang X, Ye C, et al. Satisfactory degree of the community health services in Chengdu and its influential factors. Yu Fang Yi Xue Qing Bao Za Zhi 2012;28:193-6.

12 Guan J, Li J, Liang WN. Actuality of Inhabitant's satisfaction about service in medical institutions. Zhongguo Quan Ke Yi Xue 2006;13:1050-3.

13 Chen MY, Chen MC. The practical value of three-level management mode of comprehensive prevention and treatment of hypertension in community. Shenzhen Zhong Xi Yi Jie He Za Zhi 2019;29:169-71.

14 Tao MY, Zhang YY, Shen Y, et al. Effects of Specialist's Involvement in Community Hypertension Management. Zhongguo Chu Ji Wei Sheng Bao Jian 2013;27:25-6.

15 Whelton PK, Carey RM, Aronow WS, et al. 2017 ACC/AHA/AAPA ABC/ACPM/AGS/APhA/ASH/ASPC/NMA/PCNA guideline for the prevention, detection, evaluation, and management of high blood pressure in adults: a report of the American College of Cardiology/ American heart association Task force on clinical practice guidelines. Hypertension 2018;71:e13-115.

16 Omboni S, Gazzola T, Carabelli G, et al. Clinical usefulness and cost effectiveness of home blood pressure telemonitoring: meta-analysis of randomized controlled studies. $J$ Hypertens 2013;31:455-68.

17 Nolan RP, Feldman R, Dawes M, et al. Randomized controlled trial of E-Counseling for hypertension: reach. Circ Cardiovasc Qual Outcomes 2018;11:e004420.

18 Alessa T, Hawley MS, Hock ES, et al. Smartphone Apps to support self-management of hypertension: review and content analysis. JMIR Mhealth Uhealth 2019;7:e13645.

19 Chan A-W, Tetzlaff JM, Altman DG, et al. Spirit 2013 statement: defining standard protocol items for clinical trials. Ann Intern Med 2013;158:200-7.

20 Chan A-W, Tetzlaff JM, Gøtzsche PC, et al. Spirit 2013 explanation and elaboration: guidance for protocols of clinical trials. $B M J$ 2013;346:e7586

21 Grol R, Wensing M. Patients evaluate general/family practice. The EUROPEP instrument, center for quality of care research, Nijmegen, the Netherlands, 2000: 9-70.

22 Svarstad BL, Chewning BA, Sleath BL, et al. The brief medication questionnaire: a tool for screening patient adherence and barriers to adherence. Patient Educ Couns 1999;37:113-24.

23 Persell SD, Peprah YA, Lipiszko D, et al. Effect of home blood pressure monitoring via a smartphone hypertension coaching application or tracking application on adults with uncontrolled hypertension: a randomized clinical trial. JAMA Netw Open 2020;3:e200255.

24 Williams B, Mancia G, Spiering W, et al. 2018 ESC/ESH guidelines for the management of arterial hypertension. Eur Heart $J$ 2018;39:3021-104.

25 League $\mathrm{CH}$. Guideline for blood pressure monitoring at home in China (2019). Zhonghua Gao Xue Ya Za Zhi 2019;27:708-11.

26 Margolis KL, Asche SE, Bergdall AR, et al. Effect of home blood pressure telemonitoring and pharmacist management on blood pressure control: a cluster randomized clinical trial. JAMA 2013;310:46-56.

27 Ettehad D, Emdin CA, Kiran A, et al. Blood pressure lowering for prevention of cardiovascular disease and death: a systematic review and meta-analysis. Lancet 2016;387:957-67.

28 Mills KT, Rubinstein A, Irazola V, et al. Comprehensive approach for hypertension control in low-income populations: rationale and study design for the hypertension control program in Argentina. Am J Med Sci 2014;348:139-45.

$29 \mathrm{He} \mathrm{J}$, Irazola V, Mills KT, et al. Effect of a community health WorkerLed multicomponent intervention on blood pressure control in 
low-income patients in Argentina: a randomized clinical trial. JAMA 2017:318:1016-25.

30 Jafar TH, Gandhi M, de Silva HA, et al. A community-based intervention for managing hypertension in rural South Asia. N Engl J Med 2020;382:717-26.

31 Wang J, Zhang X, Yan QQ. The current situation of community hypertension management and prevention under the background of hierarchical medical system. Zhong Xi Yi Jie He Xin Xue Guan Bing Dian Zi Za Zhi 2019;7:26-8.

32 Zhang YS. Difficulties and suggestions on the implementation of hierarchical medical system in poor areas in Western China. Xin Xi Bu 2017;14:70-2.

33 LIU LL, ZHANG SQ. Gong f. current status of general practitioner training in Chongqing and corresponding policy recommendation. Zhongguo Quan Ke Yi Xue 2017;20:89-94.
34 Li M, Wang R, Ning C. Current situation of and rethinking on the implementation of hierarchical medical system in Shanxi Province. Zhongguo Yi Liao Guan Li Ke Xue 2016;6:10-13.

35 Liu S, Dunford SD, Leung YW, et al. Reducing blood pressure with Internet-based interventions: a meta-analysis. Can J Cardiol 2013;29:613-21.

36 Purcell R, Mclnnes S, Halcomb EJ. Telemonitoring can assist in managing cardiovascular disease in primary care: a systematic review of systematic reviews. BMC Fam Pract 2014;15:43.

37 Sayani S, Muzammil M, Saleh K, et al. Addressing cost and time barriers in chronic disease management through telemedicine: an exploratory research in select low- and middle-income countries. Ther Adv Chronic Dis 2019;10:2040622319891587. 